\title{
The ethnobotany and biogeography of wild vegetables in the Adriatic islands
}

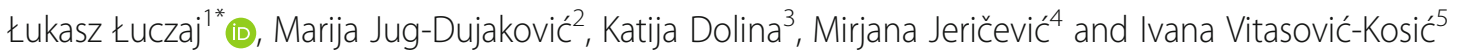

\begin{abstract}
Background: Archipelagos of islands have played an important role in shaping some of the paradigms of biology, including the theory of the evolution of species. Later, their importance in biology was further emphasised by the theory of island biogeography, which contributed to a better understanding of the shaping of species richness not only on real islands, but on isolated habitat islands as well. Although ethnobotany is a well-established discipline, patterns of knowledge about plant uses in archipelagos have never been quantitatively analysed, and the whole concept has been only briefly mentioned in the ethnobiological context.

The aim of our study was to record which taxa of wild vegetables have been consumed in the Adriatic islands and to establish if such variables as island size, population size, flora or its isolation are correlated with the number of wild vegetables used.

Methods: We interviewed 225 people (15 from each island).

Results: Altogether, the use of 89 species of wild vegetables has been recorded. The largest number of wild vegetables is eaten on the islands of Korčula, Vis and Šolta, and the lowest on Ugljan, Cres and Dugi Otok. The studied independent variables had a small and statistically not significant effect on the wild vegetable list length. The most visible effect was an increasing trend from north-west to south-east, overrunning the typical biogeographical island patterns. Moreover, one of the large and well-populated islands, Korčula, showed an 'unusually' high level of wild vegetable use. We hypothesise that the current use of so many species on this island has been maintained by the inhabitants' awareness that they are the holders of relic knowledge, an awareness reiterated by ethnographic and popular publications, as well as a strong history of famine. The most interesting edible species used in the Adriatic islands are Bunium alpinum, Cytinus hypocystis (both mainly on Pašman), Lotus edulis (on Vis) and Posidonia oceanica (on Vis and Korčula).

Conclusions: The recorded relationships between the demographic and geographical features of the islands were statistically not significant. We assume that cultural and historical factors diversifying the use of plants in particular islands are stronger than the above-mentioned measurable variables.
\end{abstract}

Keywords: Wild edible plants, Wild food plants, Ethnobiology, Leafy vegetables, Mediterranean diet

\section{Background}

Archipelagos of islands have played an important role in shaping some of the paradigms of biology, including the theory of the evolution of species created by Charles Darwin and Alfred Russel Wallace [1]. The former biologist developed it by the study of the features of closely related species in the Galapagos Islands and the latter by the research in the islands of present-day Indonesia. Later, their importance in biology was further emphasised by the

\footnotetext{
* Correspondence: lukasz.luczaj@interia.pl

${ }^{1}$ Department of Botany, Faculty of Biotechnology, University of Rzeszów, ul.

Pigonia 1, 35-310 Rzeszów, Poland

Full list of author information is available at the end of the article
}

theory of island biogeography created by Robert MacArthur and Edward O. Wilson [2]. This theory helps us understand the shaping of species richness not only on real islands, but on isolated habitat islands as well. Although ethnobotany is a well-established discipline, patterns of knowledge about plant uses in archipelagos have never been quantitatively analysed. The whole concept has been only briefly mentioned in the ethnobiological context [3], in spite of the fact that several ethnobotanical studies have qualitatively compared the uses of plants on groups of islands, particularly in Polynesia (e.g. [4, 5]). In our paper, we look at the species richness of wild

(c) The Author(s). 2019 Open Access This article is distributed under the terms of the Creative Commons Attribution 4.0 International License (http://creativecommons.org/licenses/by/4.0/), which permits unrestricted use, distribution, and 
vegetables used by the inhabitants of the 15 largest Adriatic islands in Croatia.

The reason island biogeography theory has not been tested in ethnobotany stems from the extreme complexity of the relationship between humans and plants. For instance, two communities using similar resources and living in the same or neighbouring areas may differ in plant use [6]. Moreover, humans migrate, and it would take very isolated islands and low technology to keep most human individuals from ever leaving their ancestral island. We should, however, keep in mind that living on islands might sometimes limit migration and reduce the exchange of human knowledge. The communities on the Eastern Adriatic Islands in Dalmatia, Croatia, which are the object of our study, have been extensively studied anthropologically (e.g. [7-10]). The people living on islands more remote from the coast display an unusually high degree of genetic isolation, endogamy and inbreeding, and even now, in the times of tourism, the percentage of indigenous island population varies between 70 and 98\% [10]. Thus, most inhabitants, even if they have travelled or lived outside the Adriatic islands, are usually associated with one island within the researched area. The aim of our study was to find out whether island characteristics had some influence on plant use patterns.

The area of our study covered the Croatian islands of the Adriatic coast. Of the 718 islands, only 47 are inhabited, in the sense that at least one person resides on that island [11]. However, many of those 'permanent' inhabitants are people who have emigrated to the mainland and draw tax benefits from being registered as island inhabitants: they visit the island only during summer, or even only every few years. Thus, the number of year-round inhabitants is well under half of the official total. The net population growth in most of the islands is negative, and the population of the islands has declined by $30 \%$ since a century ago [12].

Most of the larger Croatian islands have had their vascular floras described in detail [13, 14] and have been the subjects of biogeographical analyses [13].

We chose wild vegetables as the studied domain of knowledge. Their use used to be widespread in Mediterranean agroecosystems but is now declining due to changes in modern diets and lifestyles and the intensification of agriculture [15-18]. This also holds true for a few of the coastal areas on mainland Croatia and Herzegovina that we studied previously, as well as the island of Krk [19-25]. Using wild vegetables can be seen as one of the typical features of the Mediterranean cuisine and lifestyle $[18,26]$, and it has been highlighted that the use of numerous species of wild vegetables is more common in the south than in the temperate parts of central and northern Europe.
Up until recently, the gathering of wild vegetables had mainly been a domain of traditional knowledge passed down within families, little-influenced by literature, in contrast to ethnomedicinal knowledge, which is highly influenced by old and new texts and other media [27]. For local inhabitants, wild vegetables are a well-defined cognitive domain, and, according to our observations, the distinction between wild and domesticated greens is clearer than in the case of fruits.

The aim of the study was:

1. To record which taxa of wild vegetables have been consumed in the Adriatic islands

2. To establish if such variables as island size, population size, flora or its isolation are correlated with the number of wild vegetables used

We made a hypothesis that the length of the total wild vegetable list per island, as well as the median number of species per informant, is positively correlated with:

1. The number of species reported in the floras of specific islands. The link between the flora and plant use is obvious: the more species available, the more likely it is that more species are used.

2. The area of the island. A larger area within which interviews were carried out meant a larger chance for different species to be found as well as a smaller similarity in village traditions due to the larger physical distance between villages.

3. The number of inhabitants. The more people live on the island, the more exchange of knowledge is likely to happen and there are more knowledge holders.

4. The proximity of mainland (i.e. is negatively correlated with the distance from the mainland of Croatia). We assumed that in less isolated islands, whose inhabitants have more social contacts with the mainland, there is more opportunity for the exchange of knowledge.

The hypotheses no. 2 and 4 are directly testing the island biogeography theory [2] and no. 1 and 3 result from it indirectly.

\section{Methods}

We selected the 15 largest islands, those with an area $>40$ $\mathrm{km}^{2}$ (Table 1, Fig. 1). The study was performed between 2013 and 2018, with most interviews conducted in 2016 and 2017, in seasons when wild vegetables can be found (spring or autumn). We applied the classic methods of ethnobotany [28-31]: in-depth semi-structured interviews starting from freelisting and supplemented, if possible, by walks around the places where the respondents gathered 
Table 1 Basic island statistics

\begin{tabular}{lccccccc}
\hline & $\begin{array}{l}\text { No. of wild } \\
\text { vegetables }\end{array}$ & $\begin{array}{c}\text { Median no. of } \\
\text { vegetables }\end{array}$ & Area $\left(\mathrm{km}^{2}\right)$ & Population & Flora & Longitude $\left(^{\circ}\right.$ E) & Isolation (minimum km distance from mainland) \\
\hline Brač & 30 & 9 & 395 & 13,956 & 750 & 16.66 & 5 \\
Cres & 18 & 4 & 406 & 3079 & 1250 & 14.39 & 5 \\
Dugi Otok & 19 & 4 & 113 & 1655 & 540 & 15.03 & 16 \\
Hvar & 22 & 9 & 297 & 11,077 & 1046 & 16.8 & 4 \\
Korčula & 46 & 16 & 271 & 15,522 & 858 & 16.93 & 1 \\
Krk & 29 & 8 & 405 & 19,383 & 1170 & 14.61 & 0.8 \\
Lastovo & 21 & 8 & 41 & 792 & 678 & 16.87 & 26 \\
Lošinj & 26 & 8 & 74 & 7587 & 1300 & 14.43 & 29 \\
Mljet & 30 & 7 & 98 & 1088 & 712 & 17.55 & 8 \\
Pag & 31 & 9 & 284 & 9059 & 650 & 15.04 & 0.4 \\
Pašman & 26 & 7 & 60 & 2845 & 629 & 15.34 & 2 \\
Rab & 24 & 7 & 86 & 9328 & 800 & 14.77 & 2 \\
Šolta & 33 & 11 & 58 & 1700 & 267 & 16.31 & 15 \\
Ugljan & 18 & 8 & 51 & 6049 & - & 15.17 & 4 \\
Vis & 39 & 12 & 90 & 3445 & 598 & 16.16 & 43 \\
\hline
\end{tabular}

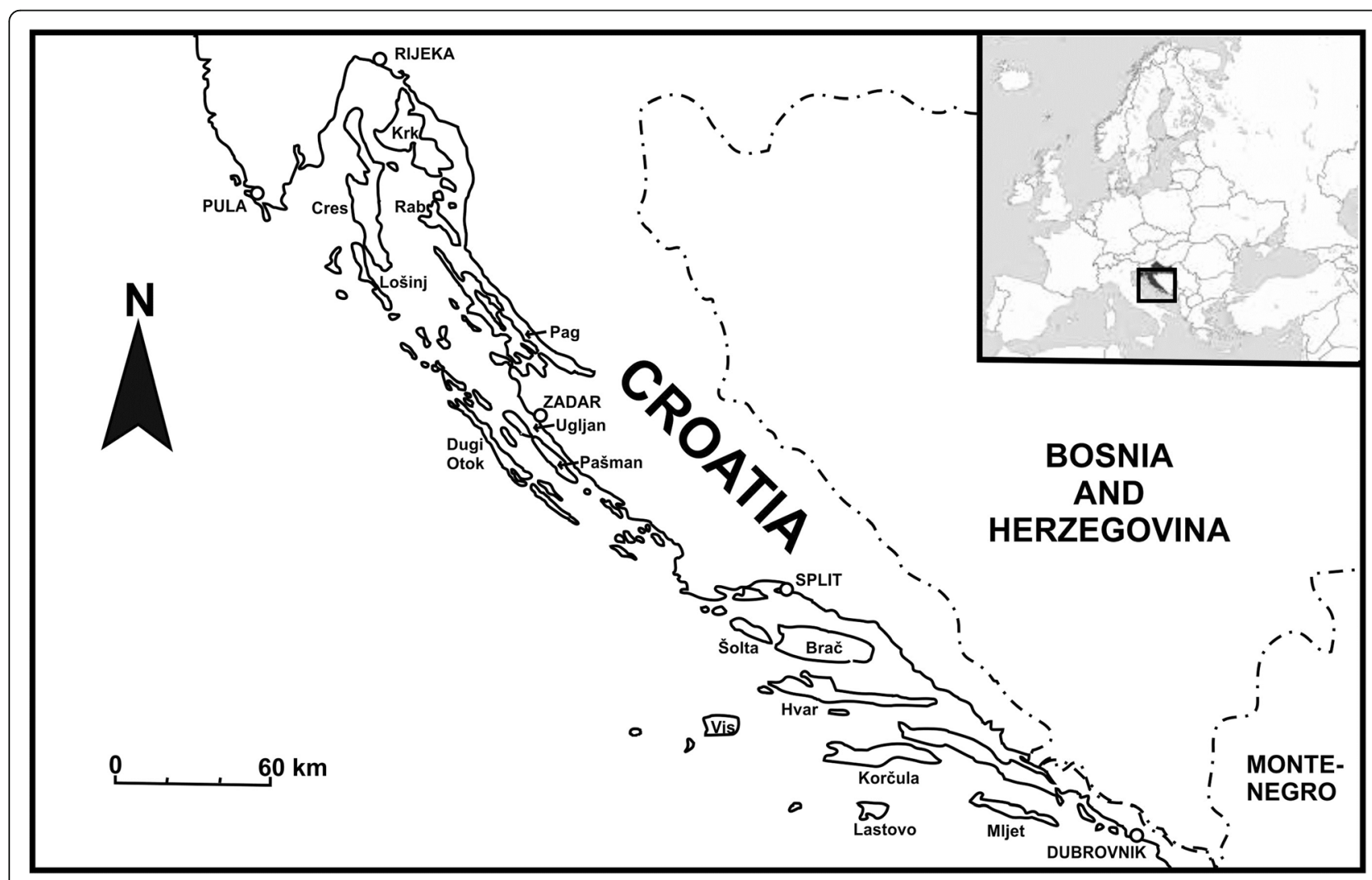

Fig. 1 Map of the Adriatic Sea and southern Croatia showing the studied islands (normal font) and major cities (in capitals) 
plants and could identify the supplied names. On each island, we interviewed 15 key informants (those who know and collect wild foods) recommended by inhabitants, villages leaders, etc. Some key informants were also selected from people found working in the fields and claiming that they still collected wild food plants. The interviews were performed in Croatian, the native language of the inhabitants. The interviews concerned different aspects of plant use, but here, we present data only about wild vegetables. The general question of which 'wild vegetables' people used for food was supplemented with questions about 'edible asparagus-like plants' and wild vegetables preserved in vinegar, as some respondents tended to forget these plants when asked only about 'wild vegetables.' The category of asparagus-like plants is an emic one. It consists of plants whose young long shoots are eaten.

We made efforts to cover the whole island evenly and recruit each informant from a different village (if the number of villages on an island was over 14). The number 15 was chosen as in less populated islands, it was difficult to recruit a larger number of key informants who actively gathered wild vegetables. We interviewed more women $(65 \%)$ than men as they were usually identified by local informants as key informants; however, on each island, some male informants were also interviewed. The mean age of informants was 70 . Key informants were chosen from people who were born on the islands and had their ancestry there.

The data for most islands has never been used before in any paper, but the data for Krk forms a subset of a larger set of interviews from this island performed for the comparison of historical and present uses of wild plants [21]. From this subset, we chose the first 15 interviews, which represented 15 villages.

The number of species in the islands' floras was extracted from data gathered by Nikolić et al. [13], supplemented by the flora of Pašman [14]. The island's isolation was measured as the distance $(\mathrm{km})$ between the mainland and the part of the island closest to it. The population data were taken from the Statistical Yearbook as of 2015 [11].

Plants were identified using standard floras available in this area of Europe, including Domac's guide for the identification of Croatian flora [32], Pignatti's flora of Italy [33] and the Flora Croatica Database [34]. Plant names were updated to be consistent with the Plant List [35]. Voucher specimens were collected on the islands where they are used, usually with the assistance of the respondents. For deposition place, see the 'Availability of data and materials' section of the paper.

Statistical analysis was performed using open access PAST software [36]. The significance and strength of the relationships between variables was assessed using correlation coefficients. The normality of distribution of variables was tested with the Shapiro-Wilk test. Most variables had normal distribution. Only the variable Isolation had to be log-transformed to achieve normality and the variable Area did not become normal even after log-transformation. That is why for the latter variable, we applied the non-parametric Spearman rank correlation coefficient, whereas other variables were correlated using the parametric Pearson correlation coefficient. To visualise the similarity in wild vegetable species lists between the islands, and see whether this was associated with geographical proximity, we performed a detrended component analysis (DCA) on the species level [37]. We plotted the results of DCA on the two main axes that caused the distribution of the data to visualise potential overlap and variation in the species composition used in different islands. Another way of visualising the diversity of species composition on different islands was a numeral taxonomy dendrogram obtained by clustering. We applied the most commonly used method of clustering, i.e. unweighted pair group method with arithmetic mean (UPGMA), using Euclidean distance [38, 39].

\section{Results and discussion}

Altogether, 89 taxa of wild vegetables from 31 plant families were identified to the species or genus level (Tables 2 and 3, Fig. 2). The longest lists of taxa used were found on Korčula (46 folk taxa), Vis (39) and Šolta (33). The shortest lists were found on Ugljan (18), Cres (18) and Dugi Otok (19). Korčula also had the highest median and mean number of species listed per interview (16). The best-represented families were Asteraceae (24 species), as well as Brassicaceae (9) and Apiaceae (8).

Five taxa, i.e. Asparagus acutifolius L., Cichorium intybus L., Dioscorea communis (L.) Caddick \& Wilkin, Foeniculum vulgare Mill. and Sonchus spp., are gathered to some extent on all the islands, and the collection of Allium ampeloprasum L., Crithmum maritimum L., Diplotaxis spp., Papaver rhoeas L., Portulaca oleracea L., and Taraxacum spp. is or was practiced on all but one or two islands.

The studied patterns of wild vegetable use were relatively weak (Table 4, Fig. 3), and no significant correlations $(p<0.05)$ between independent and dependent variables were found (although a few approached the significance level). Thus, all the hypotheses can be rejected from the statistical point of view. The highest correlations were found between geographical longitude and the number of wild vegetable species, and between population size and the number of wild vegetable species. Surprisingly, the total number of species in the flora showed a negative correlation with the median number of vegetable species used. Correlations between the total number of wild vegetable species and independent variables were nearly identical, as the total number of vegetable species per island and the median number of 
Table 2 Local names of wild vegetables

\begin{tabular}{|c|c|c|c|c|}
\hline & Voucher no. & $\begin{array}{l}\text { Part } \\
\text { used }\end{array}$ & Preparation & Most common names \\
\hline \multicolumn{5}{|l|}{ Alliaceae } \\
\hline Allium ampeloprasum L. & WA0000066378 & WH & $r / c$ & $\begin{array}{c}\text { divlji luk, poriluk; also: pazduh LO, ljutica PG, lučac LO, lučić PG, RA, porić } \\
\text { BR, paric VI, purić SO, BR, puriluk SO, HV, praska ML }\end{array}$ \\
\hline $\begin{array}{l}\text { other Allium spp. (mainly } \\
\text { Allium roseum L.) }\end{array}$ & WA0000066454 & WH & $\mathrm{r} / \mathrm{c}$ & $\begin{array}{c}\text { divji luk LO, KR, BR, divlji lučić KR, SO, jutika BR, ljutica PS, lučica KR, divlji } \\
\text { češnjak KR }\end{array}$ \\
\hline \multicolumn{5}{|l|}{ Amaranthaceae } \\
\hline Amaranthus cf. retroflexus L. & ZAGR39998 & L & c & šćirenica PG, SO, štir KO, PG, RA \\
\hline Beta vulgaris L. & WA0000066322 & L & c & divlja blitva (throughout); also: dibio blitva BR, divja blitva VI, šćav KR \\
\hline Chenopodium album L. & WA0000066308 & L & c & loboda; also: lobod KR, RA \\
\hline Salsola soda L. & WA0000066392 & L & c & rosica PG \\
\hline $\begin{array}{l}\text { Sarcocornia fruticosa (L.) } \\
\text { A.J.Scott }\end{array}$ & WA0000066902 & L & c & omaga LO, smucanj RA, smucalj RA \\
\hline \multicolumn{5}{|l|}{ Apiaceae } \\
\hline Anethum graveolens $\mathrm{L}$. & WA0000066391 & L & $r / c$ & anit SO, anita PG, aniž SO, kopar LAS \\
\hline Apium graveolens $\mathrm{L}$. & WA0000066346 & L & c & šelen PG \\
\hline $\begin{array}{l}\text { Bunium alpinum Waldst. \& Kit. } \\
\text { s.l. }\end{array}$ & WA0000066917 & $\begin{array}{l}\text { Root } \\
\text { tubers }\end{array}$ & r & koprci BR, PS, koprcini PS \\
\hline Crithmum maritimum $\mathrm{L}$. & WA0000066324 & $L$ & $\mathrm{~m}$, also c & $\begin{array}{c}\text { motar/matar; also: petrovac PG, DO, šćulac LO, ščulac PG, šćirenica KR, } \\
\text { trova od mora VI }\end{array}$ \\
\hline Daucus carota $\mathrm{L}$. & WA0000066462 & L & c & divlja mrkva; also mrkurela LA, mrkviej BR \\
\hline Foeniculum vulgare Mill. & WA0000066401 & L & $r / c$ & $\begin{array}{c}\text { komorač } \mathrm{LO}, \mathrm{DO}, \mathrm{KO}, \mathrm{ML}, \mathrm{VI} \text {, koromac LO, VI, koromač } \mathrm{BR}, \mathrm{CR} \text {, DO, HV, KO, } \\
\mathrm{KR}, \mathrm{LO}, \mathrm{PG}, \mathrm{PS}, \mathrm{RA}, \mathrm{SO}, \mathrm{UG}, \mathrm{VI} \text {, kromač KR, morač KO, ML, LA }\end{array}$ \\
\hline Smyrnium olusatrum L. & WA0000066377 & L & c & divlji selen LA, postolažina LA, postoložena LA \\
\hline Tordylium apulum L. & WA0000066382 & $L$ & c & lembrc KO, vrati muž KO \\
\hline \multicolumn{5}{|l|}{ Araceae } \\
\hline Arum italicum Mill. & WA0000066915 & $L^{a}$ & $\mathrm{lb}$ & $\begin{array}{c}\text { arum CR, gujino zelje SO, kozlac LO, štarkavac CR, strtok KR, zminac DO, } \\
\text { žuminac VI }\end{array}$ \\
\hline \multicolumn{5}{|l|}{ Asparagaceae } \\
\hline Asparagus acutifolius L. & WA0000066368 & $\mathrm{SH}$ & $c / r$ & šparoga, sparoga (throughout); also: asparadži CR, šparuga LO, šporovi CR \\
\hline Asparagus officinalis $\mathrm{L}$. & WA0000066906 & $\mathrm{SH}$ & $c / r$ & pitoma šparoga CR \\
\hline Leopoldia comosa (L.) Parl. & WA0000066916 & WH & c & fratar KR \\
\hline Ornithogalum sp. & & WH & $c$ & - \\
\hline Ruscus aculeatus L. & WA0000066369 & $\mathrm{SH}$ & c & $\begin{array}{c}\text { fraterska šparožina } K R \text {, kataroška } K R \text {, piturožka } R A \text {, pundži topo } C R \text {, rakže } \\
M L \text {, koštrika } M L \text {, sjeskavica } L A \text {, veprina } K R, L O, M L \text {, veprinac } L O\end{array}$ \\
\hline \multicolumn{5}{|l|}{ Asphodelaceae } \\
\hline Asphodelus aestivus Brot. & WA0000066433 & $\begin{array}{l}\text { Root } \\
\text { tubers }^{\mathrm{a}}\end{array}$ & $\mathrm{lb}$ & brden LO, cefarnjok VI \\
\hline \multicolumn{5}{|l|}{ Asteraceae } \\
\hline Bellis sp. & & WH & $c$ & tratinčica $\mathrm{KO}, \mathrm{VI}$ \\
\hline Carduus pycnocephalus L. & WA0000071128 & L & c & $\begin{array}{c}\text { drača SO, ošebad KO, osjak KO, oslobod VI, sikavac RA, sikavec PG, sikavica } \\
\text { DO }\end{array}$ \\
\hline Chondrilla juncea $\mathrm{L}$. & WA0000071121 & L & c & tavka PG \\
\hline Cichorium intybus L. & WA0000066320 & L & $\mathrm{r} / \mathrm{c}$ & $\begin{array}{l}\text { žutenica/žutinica/žutjenica (Dalmatian Islands); divlji radič/divlji radić } \\
\text { (Kvarner Islands, LA, ML) }\end{array}$ \\
\hline Crepis dioscoridis $\mathrm{L}$. & WA00000 & L & $r / c$ & šćupej KO, žutinica KO, gorčica BR, gorcik VI, gorčik HV \\
\hline Crepis rubra L. & WA0000066436 & L & c & šćupej KO, šćjuper BR, šćuperuša BR \\
\hline Crepis sancta (L.) Babc. & ZAGR9316 & $\mathrm{L}$ & $r / c$ & maslačak KR, RA, divlji radić RA, žutenica čupava KR \\
\hline
\end{tabular}


Table 2 Local names of wild vegetables (Continued)

\begin{tabular}{|c|c|c|c|c|}
\hline & Voucher no. & $\begin{array}{l}\text { Part } \\
\text { used }\end{array}$ & Preparation & Most common names \\
\hline $\begin{array}{l}\text { Helminthotheca echioides (L.) } \\
\text { Holub. }\end{array}$ & WA0000066360 & $\mathrm{L}$ & c & $\begin{array}{c}\text { hrastavica KO, krastavica PS, lipavac PS, lipavica PS, prosenjica RA, rastej } \\
\text { ML, tustoč BR, tustočel HV }\end{array}$ \\
\hline Lactuca perennis L. & & L & c & divlji špinat, modra salata BR \\
\hline Lactuca sativa L. [feral] & & L & $r / c$ & pičola SO, HV, loćika KO \\
\hline Lactuca serriola L. & WA0000066412 & L & c & divlja salata; also: gorka salata BR, pasja salata VI \\
\hline $\begin{array}{l}\text { Lactuca viminea (L.) J. Presl \& } \\
\text { C.Presl }\end{array}$ & WA0000071123 & L & c & gnjaška KO, nastriženica VI \\
\hline Leontodon tuberosus L. & WA0000066329 & L & c & $\begin{array}{l}\text { korenjaška KO, also: grglava BR, lavji zub } \mathrm{ML} \text {, podparuša } \mathrm{ML} \text {, ugrin glava } \\
\qquad \mathrm{KO} \text {, undrglava } \mathrm{KO}\end{array}$ \\
\hline Reichardia picroides (L.) Roth & WA0000066328 & L & $r / c$ & $\begin{array}{c}\text { antačola } R A \text {, natančola } L O \text {, } R A \text {, ratančola } R A \text {, barbaruša } K R \text {, berbečica } P G \text {, } \\
\text { b(e) rberuša } D O, P G \text {, beršaka } P G \text {, bršača } L O \text {, bršljaka } P G, M L \text {, brusača } K R \text {, } \\
\text { dušica } B R \text {, SO, iglica } \cup G \text {, jagla } L O \text {, jaglac } L O \text {, jogula } L O \text {, marta duha } B R \text {, } \\
\text { matederica VI, materduh } H R \text {, materduha } B R \text {, HV, materinduh } H V \text {, } \\
\text { matuderica } V I \text {, mojčinduh } H V \text {, slaška/slačka } K O \text {, sladić } M L \text {, tavka } L A\end{array}$ \\
\hline $\begin{array}{l}\text { Rhagadiolus stellatus (L.) } \\
\text { Gaertn. }\end{array}$ & WA0000066445 & L & c & kokošinja guzica, kokošinja guzica KO \\
\hline Scolymus hispanicus L. & WA0000066345 & L & c & $\begin{array}{c}\text { brbeč PS, bremečica PG, brisača KR, brmeč KR, oščibod VI, sikavac PG, } \\
\text { sisavica KO, skolub KO }\end{array}$ \\
\hline Scorzonera laciniata Jacq. & WA0000071122 & L & c & $\begin{array}{c}\text { kozja brada HV, BR, KO, SO, kozja broda BR, kozjo brada VI, kozjo broda VI, } \\
\text { kuzjo brada VI, kušnja broda VI }\end{array}$ \\
\hline Silybum marianum (L.) Gaertn. & WA0000066349 & L & c & beli trn KR \\
\hline Sonchus spp. & & & & $\begin{array}{c}\text { blešnjak LO, bliješnjak } L O, C R \text {, blišnjak } L O \text {, blješnjak LO, blišnjak LO, PS, } \\
\text { čepčeg/čevčeg ML, kostreč LA, kostric VI, kostrić/kostrič BR, HV, KO, LA, VI, } \\
\text { kostriš DO, LO, UG, mišnjak PS, UG, mlič/mlić PG, mličac DO, mličak UG, } \\
\text { mličnjak DO, PG, UG, mlišnjak UG, ostak/ostek CR, KR, sinjorac PG, RA, } \\
\text { špilišor KR, šušak/sušak SO }\end{array}$ \\
\hline Sonchus asper (L.) Hill & WA0000066913 & L & c & \\
\hline $\begin{array}{l}\text { Sonchus asper subsp. } \\
\text { glaucescens (Jord.) Ball ex } \\
\text { Ball }\end{array}$ & WA0000066912 & L & c & \\
\hline Sonchus oleraceus L. & WA0000066305 & L & c & \\
\hline Taraxacum sp. & WA0000066372 & L & $\mathrm{r} / \mathrm{c}$ & $\begin{array}{l}\text { maslačak (throughout), also: paric VI, retkozuba KR, žutenica KR, žutenjak } \\
\text { PS, žutinica KO, zlatenca LO }\end{array}$ \\
\hline Tragopogon porrifolius L. & WA0000066426 & L & c & kozja brada (throughout), červej BR, kužjo brada VI \\
\hline $\begin{array}{l}\text { Urospermum picroides (L.) Scop. } \\
\text { ex F.W.Schmidt }\end{array}$ & WA0000066304 & L & c & $\begin{array}{l}\text { cistacil VI, cistocel VI, lipavica PS, loćika KO, plještika ML, pješti guzica, } \\
\text { tustocel VI, tustočen BR, tustočina BR, tutošć BR, tustočel HV, kostočel KO }\end{array}$ \\
\hline \multicolumn{5}{|l|}{ Boraginaceae } \\
\hline Borago officinalis L. & WA0000066357 & L & c & borač LO, boražina LO, buražina SO, buražija KO, krastavac ML \\
\hline Echium italicum L. & WA0000066340 & L & c & - \\
\hline \multicolumn{5}{|l|}{ Brassicaceae } \\
\hline Bunias erucago L. & WA0000066909 & L & c & pakoleć $\mathrm{ML}$, šurlin $\mathrm{KO}$ \\
\hline Calepina irregularis (Asso) Thell. & WA0000066416 & L & c & šurlin $\mathrm{KO}$ \\
\hline $\begin{array}{l}\text { Capsella bursa-pastoris (L.) } \\
\text { Medik. }\end{array}$ & WA0000066371 & L & c & prskavica LA, rosomač KO, ščupic SO, šurlin KO \\
\hline Diplotaxis spp. & & & & divlja riga (throughout) \\
\hline Diplotaxis muralis (L.) DC. & WA0000066313 & L & $r / c$ & \\
\hline Diplotaxis tenuifolia (L.) DC. & WA0000066337 & L & $r / c$ & \\
\hline Eruca vesicaria (L.) Cav. & WA0000066491 & L & r & divlja riga PS \\
\hline Nasturtium officinale L. & WA0000066343 & L & $r / c$ & kreš PG, kriš KR \\
\hline Raphanus raphanistrum L. s.I. & WA0000071107 & $\mathrm{L}$ & $c$ & divlja rodakva SO, divlja repa SO, divlja rokva PS \\
\hline
\end{tabular}


Table 2 Local names of wild vegetables (Continued)

\begin{tabular}{|c|c|c|c|c|}
\hline & Voucher no. & $\begin{array}{l}\text { Part } \\
\text { used }\end{array}$ & Preparation & Most common names \\
\hline Sisymbrium officinale (L.) Scop. & WA0000066418 & L & c & drozguja KO \\
\hline \multicolumn{5}{|l|}{ Capparaceae } \\
\hline Capparis orientalis Veill. & WA0000066334 & Buds & $\mathrm{m}$ & kapar (throughout) \\
\hline \multicolumn{5}{|l|}{ Caryophyllaceae } \\
\hline Stellaria media $\mathrm{L}$. & WA0000066359 & L & c & miš(j) akinja KO \\
\hline Silene latifolia Poir. & WA0000066393 & L & c & škripac SO, škripavica PS \\
\hline Silene vulgaris (Moench) Garcke & WA0000071139 & L & c & učjak PG, uš(I) jak PG, uvce CR, pušina LO \\
\hline \multicolumn{5}{|l|}{ Convolvulaceae } \\
\hline $\begin{array}{l}\text { Convolvulus arvensis } L \text {. and } \\
\text { possibly other species from the } \\
\text { genus }\end{array}$ & ZAGR40001 & L & c & slak ML, zlak KO, KR, slačica KO \\
\hline \multicolumn{5}{|l|}{ Cytinaceae } \\
\hline Cytinus hypocistis (L.) L. & $\begin{array}{l}\text { Protected } \\
\text { species }\end{array}$ & $\mathrm{FL}+\mathrm{L}$ & $r$ & prasica PS, kokošica DO \\
\hline \multicolumn{5}{|l|}{ Dioscoreaceae } \\
\hline $\begin{array}{l}\text { Dioscorea communis (L.) } \\
\text { Caddick \& Wilkin }\end{array}$ & ZAGR39307 & $\mathrm{SH}$ & c & bljušć, bljušt, blušć, blušt; also: kuke ML, kukolj/kukelj PS, UG \\
\hline \multicolumn{5}{|l|}{ Dipsacaceae } \\
\hline $\begin{array}{l}\text { Knautia integrifolia (Honck. ex } \\
\text { L.) Bertol. }\end{array}$ & ZAGR39815 & L & c & rešetnica KR \\
\hline \multicolumn{5}{|l|}{ Euphorbiaceae } \\
\hline Mercurialis annua L. & WA0000066409 & L & c & prajc Vl, prajca VI \\
\hline \multicolumn{5}{|l|}{ Fabaceae } \\
\hline Lotus edulis L. & WA0000066450 & IF & r & golubinjica VI, gominjac VI \\
\hline $\begin{array}{l}\text { Pisum sativum subsp. elatius } \\
\text { (M.Bieb.) Asch. \& Graebn. }\end{array}$ & WA0000071112 & $\mathrm{SH}, \mathrm{IF}$ & r & divlji biž Vl \\
\hline Robinia pseudoacacia L. & WA0000066466 & $\mathrm{FL}$ & $\mathrm{r} / \mathrm{c}$ & akacija, akacia, drača PG, RA, ščavljak ŠO \\
\hline Vicia narbonensis L. & WA0000071113 & $\mathrm{SH}, \mathrm{IF}$ & r & divlji bob VI \\
\hline \multicolumn{5}{|l|}{ Geraniaceae } \\
\hline Erodium cicutarium L. & WA0000071137 & L & $c$ & iglica KO, PS \\
\hline \multicolumn{5}{|l|}{ Malvaceae } \\
\hline Malva sylvestris $\mathrm{L}$. & WA0000066400 & L & c & sljez VI, sirćić PG \\
\hline \multicolumn{5}{|l|}{ Papaveraceae } \\
\hline Papaver rhoeas $\mathrm{L}$. & WA0000066381 & L & $r / c$ & mak, also: papaver VI, ugor glova VI \\
\hline \multicolumn{5}{|l|}{ Plantaginaceae } \\
\hline Plantago spp. & & & & trputac DO, KR, PS, VI, lokvar KR, trbušac KR \\
\hline Plantago lanceolata $\mathrm{L}$. & ZAGR39306 & L & c & \\
\hline Plantago major L. & ZAGR39699 & L & c & \\
\hline Plantago media $\mathrm{L}$. & ZAGR39712 & L & c & \\
\hline \multicolumn{5}{|l|}{ Poaceae } \\
\hline Avena sterilis $\mathrm{L}$. & WA0000066925 & L & r & sviralica DO \\
\hline \multicolumn{5}{|l|}{ Polygonaceae } \\
\hline Rumex pulcher $\mathrm{L}$. & ZAGR39692 & L & c & kiselica KR, PG, šćav, divlja blitva KR \\
\hline Rumex sp. & & L & c & kravlja riljica PG \\
\hline \multicolumn{5}{|l|}{ Portulacaceae } \\
\hline Portulaca oleracea L. & WA0000066314 & $\mathrm{L}$ & $r / c$ & tucanj $\mathrm{DO}, \mathrm{PS}, \mathrm{RA}, \mathrm{UG}$, SO, tušć $\mathrm{BR}$, HV, UG VI, tušt $\mathrm{BR}, \mathrm{CR}, \mathrm{HV}, \mathrm{KO}, \mathrm{LO}, \mathrm{PG}$, \\
\hline
\end{tabular}


Table 2 Local names of wild vegetables (Continued)

\begin{tabular}{|c|c|c|c|c|}
\hline & Voucher no. & $\begin{array}{l}\text { Part } \\
\text { used }\end{array}$ & Preparation & Most common names \\
\hline & & & & SO, Vl; also: roškan BR, tušanj SO, tustoč BR, HV \\
\hline \multicolumn{5}{|l|}{ Posidoniaceae } \\
\hline Posidonia oceanica (L.) Delile & WA0000066903 & $\begin{array}{c}\text { Basal } \\
\text { part }\end{array}$ & r & valiga $\mathrm{KO}$, vlasnica $\mathrm{VI}$, vlasinica $\mathrm{VI}$ \\
\hline \multicolumn{5}{|l|}{ Ranunculaceae } \\
\hline Clematis vitalba $\mathrm{L}$. & WA0000066476 & $\mathrm{SH}$ & c & pavitina LO, škrabutina $M L$, škrebut $C R$, tertina/trtina/trta $K R$ \\
\hline \multicolumn{5}{|l|}{ Rosaceae } \\
\hline Rubus ulmifolius Scott. & ZAGR39711 & $\mathrm{SH}$ & & drača ML, kupina VI \\
\hline \multicolumn{5}{|l|}{ Rubiaceae } \\
\hline Theligonum cynocrambe L. & WA0000066437 & L & c & kokošja jetrica BR \\
\hline \multicolumn{5}{|l|}{ Smilacaceae } \\
\hline Smilax aspera $\mathrm{L}$. & WA0000066325 & $\mathrm{SH}$ & c & $\begin{array}{l}\text { tetevika BR, KO, ML, tetivika LO, tetovica } \mathrm{HV} \text {, totovika/tutuvika SO, tutuvica } \\
\qquad \mathrm{VI} \text {, jarika } \mathrm{PG}\end{array}$ \\
\hline \multicolumn{5}{|l|}{ Urticaceae } \\
\hline Parietaria judaica L. & WA0000066338 & L & c & šćurenica KR, crkvina KO, SO, šćirenica LO, RA \\
\hline Urtica spp. & & & & $\begin{array}{c}\text { kopriva } M L, R A, S O, U G, L O, V I, S O, K R, L A \text {; also: ožeguja } C R \text {, ožigulja } C R \text {, } \\
\text { už(e) gavica KR, žegavica PS, žiguja, ortika CR, žigavica CR, KR, pokriva KR, } \\
\text { žgavica, KR }\end{array}$ \\
\hline Urtica dioica L. & WA0000066481 & L & c & \\
\hline Urtica pilulifera L. & WA0000066441 & L & c & \\
\hline Urtica urens L. & WA0000066423 & L & c & \\
\hline \multicolumn{5}{|l|}{ Violaceae } \\
\hline Viola odorata L. & WA0000066363 & $\mathrm{FL}$ & $r$ & ljubičica LO, ljubica KO \\
\hline
\end{tabular}

Part used-L leaf, WH whole, IF immature fruits, FL flowers, SH asparagus-like shoots (young vegetative shoots, especially their top part) Preparation $-r$ raw, $c$ cooked, l $b$-long baking or boiling, $m$ marinated in vinegar

The codes consisting of two letters (in the last column) indicate the first two letters of the name of the studied island, except DO for Dugi Otok, PG for Pag, PS for Pašman and SO for Šolta

ased only until the mid-twentieth century

vegetables listed were highly correlated. The island's degree of isolation from the mainland and its area seemed to have negligible effects on the median wild vegetable number listed. We must bear in mind that the above-discussed correlations are statistically not significant. The question rises whether the results would be significant or different if a larger number of respondents were studied. The answer is probably 'not', as we think that the 15 interviews we did for each island were very representative. This is supported by data from two islands from which we have more interviews. In the largest and most populated island, Krk, 55 interviews were conducted altogether [21] and 33 species of wild vegetables were recorded, whereas in the first 15 interviews selected for this study, 29 were found. However, the effect of some of the independent variables (area, population, flora) might have been stronger if islands smaller than $40 \mathrm{~km}^{2}$ had been included.

Surprisingly, geographical location expressed by longitude was most strongly correlated with wild vegetable species richness. This indicates that a larger scale pattern of increasing wild vegetable 'popularity' going from the northeastern Adriatic southeastwards towards Dubrovnik is stronger than island biogeography effects. A similar southeastward pattern was earlier detected for the richness of wild vegetables sold in the markets along the main coast of Croatia [19]. The spatial distribution of islands on the two main axes of DCA analysis corresponded to some extent to their geographical position. All the central Dalmatian islands (i.e. Šolta, Brač, Hvar and Vis) created one cluster together with Korčula and Pašman (which is the closest to them from all the Zadar Archipelago islands). Most islands of the Zadar Archipelago (i.e. north Dalmatian islands-Pag, Dugi Otok and Ugljan) were clustered together with the islands of the Kvarner Archipelago (Cres, Krk and Rab). Krk and the central Dalmatian Vis were most distant from other islands and formed two opposite sides of the diagram (Fig. 4).

The dendrogram from UPGMA clustering (Fig. 5) shows similar results to the DCA analysis. Here, all the central Dalmatian islands (i.e. Šolta, Brač, Hvar and Vis) 
Table 3 The diversity of wild vegetables on different islands with the number of interviews in which they were listed (15 interviews were performed in each island)

\begin{tabular}{|c|c|c|c|c|c|c|c|c|c|c|c|c|c|c|c|c|}
\hline & $\overline{A L L}$ & Brač & Cres & $\begin{array}{l}\text { Dugi } \\
\text { Otok }\end{array}$ & Hvar & Korčula & Krk & Lastovo & Lošinj & Mljet & Pag & Pašman & Rab & Šlta & Ugljan & Vis \\
\hline $\begin{array}{l}\text { The total number of folk species in } 15 \\
\text { interviews }\end{array}$ & & 30 & 18 & 19 & 22 & 46 & 29 & 21 & 26 & 30 & 31 & 26 & 24 & 33 & 18 & 39 \\
\hline Allium ampeloprasum $\mathrm{L}$. & 142 & 14 & & 5 & 15 & 13 & 2 & 11 & 9 & 9 & 12 & 13 & 3 & 14 & 7 & 15 \\
\hline other Allium spp. (mainly Allium roseum L.) & 6 & 1 & & & & & 2 & & 1 & & & 1 & & 1 & & \\
\hline Amaranthus cf. retroflexus L. & 6 & & & & & 2 & y & & & & 2 & & 1 & 1 & & \\
\hline Anethum graveolens $\mathrm{L}$. & 11 & 1 & & & & & & 3 & & & 2 & & & 5 & & \\
\hline Apium graveolens $\mathrm{L}$. & 1 & & & & & & & & & & 1 & & & & & \\
\hline Arum italicum Mill. & 7 & & 2 & 1 & & & 1 & & 1 & & & & & 1 & & 1 \\
\hline Asparagus acutifolius L. & 173 & 11 & 13 & 6 & 7 & 9 & 14 & 15 & 14 & 11 & 13 & 10 & 12 & 13 & 13 & 12 \\
\hline Asparagus officinalis $\mathrm{L}$. & 1 & & 1 & & & & & & & & & & & & & \\
\hline Asphodelus aestivus Brot. & 2 & & & & & & & & 1 & & & & & & & 1 \\
\hline Avena sterilis $\mathrm{L}$. & 1 & & & 1 & & & & & & & & & & & & \\
\hline Bellis sp. & 2 & & & & & 1 & & & & & & & & & & 2 \\
\hline Beta vulgaris $\mathrm{L}$. & 28 & 1 & 2 & 1 & & 1 & y & 4 & 12 & 2 & & 1 & & & 3 & 1 \\
\hline Borago officinalis L. & 13 & & & & & 1 & & & 10 & 1 & & & & 1 & & \\
\hline Bunias erucago L. & 2 & & & & & 1 & & & & 1 & & & & & & \\
\hline Bunium alpinum Waldst. \& Kit. s.l. & 10 & 1 & & & & & & & & & & 8 & & & 1 & \\
\hline Calepina irregularis (Asso) Thell. & 2 & & & & & 2 & & & & & & & & & & \\
\hline Capparis orientalis Veill. & 43 & 5 & & & 6 & 4 & & 6 & 2 & 1 & 1 & & 1 & 8 & 1 & 8 \\
\hline Capsella bursa-pastoris (L.) Medik. & 4 & & & & & 2 & & 1 & & & & & & 1 & & \\
\hline Carduus pycnocephalus L. & 10 & & & 1 & & 4 & & & & & 1 & & 1 & 2 & & 1 \\
\hline Chenopodium album $\mathrm{L}$. & 13 & & & & & 1 & 1 & 1 & & & 5 & & 1 & & 1 & 3 \\
\hline Chondrilla juncea $\mathrm{L}$. & 1 & & & & & & & & & & 1 & & & & & \\
\hline Cichorium intybus L. & 138 & 3 & 2 & 7 & 11 & 6 & 5 & 13 & 6 & 14 & 14 & 14 & 10 & 9 & 14 & 10 \\
\hline Clematis vitalba $\mathrm{L}$. & 10 & & 1 & & & & 7 & & 1 & 1 & & & & & & \\
\hline $\begin{array}{l}\text { Convolvulus arvensis } L \text {. and possibly } \\
\text { other species from the genus }\end{array}$ & 3 & & & & & 1 & 1 & & & 1 & & & & & & \\
\hline Crepis dioscoridis L. & 22 & 3 & & & 8 & 3 & & & & & & & & & & 8 \\
\hline Crepis rubra L. & 5 & 2 & & & & 3 & & & & & & & & & & \\
\hline Crepis sancta (L.) Babc. & 4 & & 2 & & & 1 & y & & & & & & 1 & & & \\
\hline Crepis sp. - other species & 8 & & 3 & & 1 & & & & 1 & 1 & 1 & & 1 & & & \\
\hline Crithmum maritimum L. & 60 & 3 & 1 & 3 & 2 & 5 & 1 & 7 & 10 & 7 & 5 & & 1 & 8 & 1 & 6 \\
\hline Cytinus hypocistis (L.) L. & 6 & & & 1 & & & & & & & & 5 & & & & \\
\hline Daucus carota L. & 13 & 1 & & & & 8 & & 1 & & & 1 & 1 & & 1 & & \\
\hline $\begin{array}{l}\text { Dioscorea communis (L.) Caddick \& } \\
\text { Wilkin }\end{array}$ & 88 & 8 & 4 & 2 & 1 & 7 & 9 & 7 & 6 & 14 & 10 & 2 & 2 & 8 & 6 & 2 \\
\hline Diplotaxis spp. & 90 & 7 & 10 & 5 & 2 & 5 & 11 & & 9 & 2 & 5 & 4 & 7 & 7 & 6 & 10 \\
\hline \multicolumn{17}{|l|}{ Diplotaxis muralis (L.) DC. } \\
\hline \multicolumn{17}{|l|}{ Diplotaxis tenuifolia (L.) DC. } \\
\hline Echium italicum L. & 1 & & & & & & & & & & 1 & & & & & \\
\hline Erodium cicutarium L. & 3 & & & & & 1 & & & & & & 2 & & & & \\
\hline Eruca vesicaria (L.) Cav. & 1 & & & & & & & & & & & 1 & & & & \\
\hline unidentified Fabaceae & 2 & & & & & & 1 & & & & 1 & & & & & \\
\hline Foeniculum vulgare Mill. & 153 & 13 & 4 & 13 & 11 & 14 & 8 & 3 & 10 & 5 & 13 & 12 & 12 & 11 & 11 & 13 \\
\hline
\end{tabular}


Table 3 The diversity of wild vegetables on different islands with the number of interviews in which they were listed (15 interviews were performed in each island) (Continued)

\begin{tabular}{|c|c|c|c|c|c|c|c|c|c|c|c|c|c|c|c|c|}
\hline & ALL & Brač & Cres & $\begin{array}{l}\text { Dugi } \\
\text { Otok }\end{array}$ & Hvar & Korčula & Krk & Lastovo & Lošinj & Mljet & Pag & Pašman & Rab & Šolta & Ugljan & Vis \\
\hline $\begin{array}{l}\text { The total number of folk species in } 15 \\
\text { interviews }\end{array}$ & & 30 & 18 & 19 & 22 & 46 & 29 & 21 & 26 & 30 & 31 & 26 & 24 & 33 & 18 & 39 \\
\hline Helminthotheca echioides (L.) Holub. & 18 & 1 & & & 3 & 2 & & & & 1 & & 6 & 4 & & & $\overline{1}$ \\
\hline Knautia integrifolia (Honck. ex L.) Bertol. & 2 & & & & & & 2 & & & & & & & & & \\
\hline Lactuca perennis L. & 3 & 1 & & & & & & & & & & & & 1 & & 1 \\
\hline Lactuca sativa L. [feral] & 3 & & & & 1 & 1 & & & & & & & & 1 & & \\
\hline Lactuca serriola $\mathrm{L}$. & 18 & 2 & & 1 & 2 & 4 & & 1 & & 3 & & 2 & & 2 & & 1 \\
\hline Lactuca viminea (L.) J. Presl \& C.Presl & 6 & & & & & 5 & & & & & & & & & & 1 \\
\hline Leontodon tuberosus L. & 11 & 1 & & & & 7 & & & & 3 & & & & & & \\
\hline Lotus edulis L. & 4 & & & & & & & & & & & & & & & 4 \\
\hline Malva sylvestris $\mathrm{L}$. & 2 & & & & & & & & & & 1 & & & & & 1 \\
\hline Mercurialis annua L. & 3 & & & & & z & & & & & & & & & & 3 \\
\hline Leopoldia comosa (L.) Parl. & 1 & & & & & & 1 & & & & & & & & & \\
\hline Nasturtium officinale L. & 4 & & & & & & 1 & & & & 3 & & & & & \\
\hline Ornithogalum sp. & 1 & & & & & & & & & & & & & 1 & & \\
\hline Papaver rhoeas L. & 85 & 10 & & 5 & 10 & 6 & 4 & 3 & & 9 & 2 & 8 & 5 & 11 & 5 & 7 \\
\hline Parietaria judaica L. & 6 & & & & & 1 & 1 & & 1 & & & & 1 & 1 & & 1 \\
\hline $\begin{array}{l}\text { Pisum sativum subsp. elatius (M.Bieb.) } \\
\text { Asch. \& Graebn. }\end{array}$ & & & & & & & & & & & & & & & & 1 \\
\hline Plantago spp. & 6 & & & 1 & & & 3 & & & & & 1 & & & & 1 \\
\hline Plantago lanceolata $\mathrm{L}$. & & & & & & & & & & & & & & & & \\
\hline Plantago major $\mathrm{L}$. & & & & & & & & & & & & & & & & \\
\hline Plantago media L. & & & & & & & & & & & & & & & & \\
\hline Portulaca oleracea L. & 55 & 5 & 1 & 8 & 3 & 2 & 4 & 1 & 1 & & 2 & 3 & 4 & 7 & 9 & 5 \\
\hline Posidonia oceanica (L.) Delile & 2 & & & & & y & & & & & & & & & & 2 \\
\hline Raphanus raphanistrum L. s.l. & 5 & & & & & & & & & & & 1 & & 4 & & \\
\hline Reichardia picroides (L.) Roth & 94 & 12 & & 2 & 10 & 13 & 2 & 1 & 9 & 3 & 10 & & 7 & 11 & 4 & 10 \\
\hline Rhagadiolus stellatus (L.) Gaertn. & 1 & & & & & 1 & & & & & & & & & & \\
\hline Robinia pseudoacacia L. & 2 & & & & & & & & & & 1 & & 1 & & & \\
\hline Rubus ulmifolius L. & 2 & & & & & & & & & 1 & & & & & & 1 \\
\hline Rumex pulcher $\mathrm{L}$. & 9 & & & & & 4 & 1 & & & 1 & 2 & & & & 1 & \\
\hline Ruscus aculeatus L. & 18 & & 1 & & & & 8 & 3 & 2 & 2 & & & 2 & & & \\
\hline Salsola soda L. & 2 & & & & & & & & & & 2 & & & & & \\
\hline Sarcocornia fruticosa (L.) A.J.Scott & 8 & & & & & & & & 2 & & & & 6 & & & \\
\hline Scolymus hispanicus L. & 11 & & & & & 2 & 2 & & & & 5 & 1 & & & & 1 \\
\hline Scorzonera laciniata Jacq. & 12 & 4 & & & 1 & 4 & & & & & & 1 & & 1 & & 1 \\
\hline Silene latifolia Poir. & 9 & & 1 & & & & & & & 1 & 7 & & & & & \\
\hline Silene vulgaris (Moench) Garcke & 3 & & & & & & & & 1 & & & 1 & & 1 & & \\
\hline Silybum marianum (L.) Gaertn. & 1 & & & & & & 1 & & & & & & & & & \\
\hline Sisymbrium officinale (L.) Scop. & 2 & & & & & 1 & & & & 1 & & & & & & \\
\hline Smilax aspera L. & 10 & 1 & & & 1 & 1 & & & 1 & 2 & 1 & & & 2 & & 1 \\
\hline Smyrnium olusatrum L. & 3 & & & & & & & 3 & & & & & & & & \\
\hline Sonchus spp. & 175 & 15 & 9 & 6 & 14 & 14 & 8 & 12 & 10 & 13 & 14 & 14 & 12 & 14 & 6 & 14 \\
\hline
\end{tabular}

Sonchus asper (L.) Hill 
Table 3 The diversity of wild vegetables on different islands with the number of interviews in which they were listed (15 interviews were performed in each island) (Continued)

\begin{tabular}{|c|c|c|c|c|c|c|c|c|c|c|c|c|c|c|c|c|}
\hline & ALL & Brač & Cres & $\begin{array}{l}\text { Dugi } \\
\text { Otok }\end{array}$ & Hvar & Korčula & Krk & Lastovo & Lošinj & Mljet & Pag & Pašman & Rab & Šolta & Ugljan & Vis \\
\hline $\begin{array}{l}\text { The total number of folk species in } 15 \\
\text { interviews }\end{array}$ & & 30 & 18 & 19 & 22 & 46 & 29 & 21 & 26 & 30 & 31 & 26 & 24 & 33 & 18 & 39 \\
\hline \multicolumn{17}{|l|}{$\begin{array}{l}\text { Sonchus asper subsp. glaucescens } \\
\text { (Jord.) Ball ex Ball }\end{array}$} \\
\hline \multicolumn{17}{|l|}{ Sonchus oleraceus L. } \\
\hline Stellaria media L. & 2 & & & & & 2 & & & & & & & & & & \\
\hline Taraxacum sp. & 43 & 2 & 3 & 2 & 2 & 4 & 12 & 5 & 1 & 5 & 2 & 1 & 1 & 1 & 1 & 1 \\
\hline Theligonum cynocrambe L. & 1 & 1 & & & & z & & & & & & & & & & \\
\hline Tordylium apulum L. & 8 & & & & & 8 & & & & & & & & & & \\
\hline Tragopogon porrifolius L. & 12 & 2 & & & 2 & 2 & & & & 1 & & & & 4 & & 1 \\
\hline $\begin{array}{l}\text { Urospermum picroides (L.) Scop. ex } \\
\text { F.W.Schmidt }\end{array}$ & 25 & 3 & & & 5 & 5 & & & & 4 & & 2 & & 1 & & 5 \\
\hline Urtica spp. & 38 & 8 & 5 & & & & 10 & 4 & 2 & 1 & & 1 & 2 & 6 & 5 & 4 \\
\hline \multicolumn{17}{|l|}{ Urtica dioica L. } \\
\hline \multicolumn{17}{|l|}{ Urtica pilulifera $\mathrm{L}$. } \\
\hline \multicolumn{17}{|l|}{ Urtica urens L. } \\
\hline Vicia narbonensis L. & 1 & & & & & & & & & & & & & & & 1 \\
\hline Viola odorata L. & 2 & & & & & 1 & $y$ & & 1 & & & & & & & \\
\hline
\end{tabular}

created one cluster together with Korčula and Pašman. As this cluster also contains the islands with the strongest use of wild vegetables, we could say that this area now constitutes the core region in which knowledge is preserved in the Adriatic, whereas the 'peripheral' islands north and south of it are those where wild vegetables have been forgotten to a greater extent.

The island biogeography theory [2] states that the species diversity of islands is positively correlated with the island size and negatively correlated with its distance from the mainland. It is striking that vascular floras are negatively correlated (though again not significantly) with wild vegetable diversity. It is probably caused by the fact that most wild vegetables are ruderal weeds, which may thrive better in anthropogenic degraded habitats rather than on natural islands with better preserved (semi-) natural vegetation.

Although the wild vegetables used on the islands are very similar to those on the main coast of Croatia, their preparation differs slightly (Fig. 5). On the islands, people tend to cook the wild vegetables only for a short time or eat them raw, whereas on the mainland, the vegetable mix is often cooked for 20-30 min [19-22]. Asparagus spp. and asparagus-like plants are usually prepared separately, boiled or fried and eaten with eggs. Tender, bitter Asteraceae, such as Cichorium, Crepis and Taraxacum, as well as Diplotaxis, Portulaca and Papaver, are eaten raw or only briefly boiled. C. maritimum shoots and Capparis orientalis Veill. flower buds are marinated in home-made wine vinegar. Other species are usually mixed and boiled. Wild vegetables are often cooked with one or two potatoes and served with plenty of olive oil (Fig. 6). The mixed wild vegetables are usually called interchangeably divlje zelje (literally 'wild herbs') or mišanca (literally 'mixture'), with small phonetic variants of these names depending on the dialect of the particular settlement. For example, on the island of Rab in Palit, we recorded the name mišancija, in Banjol mišjanca, and mješanca in the town of Rab. The largest variety of names for the mixture occurs on Brač with parić in Sumartin, parež in Gornji Humac, porež in Pražnice, Pučišća and Škrip, divljač in Pražnice, poreč in Nerežišče, pareš in Bol, divjo zelje in Dračevica, Mirca and Milna, and mišancja in Gornji Humac. Around the town of Cres (island Cres), the names are divljina or the Italian word erbate. On the island of Korčula, the western part (Vela Luka and Blato) uses the name gruda and the eastern part (e.g. Čara and Žrnovo) uses the name parapač. Pakojeć is the name used on Lastovo, whereas on Mljet it is called pakoleć and podparuša. On Vis, it is called gorko/gorku zelje (literally 'bitter herb') or divjo/ divlju zelje.

Although we recorded a long list of wild vegetables used in the archipelago as a whole, the use of this category of food has dramatically declined. On some islands, such as Cres or Lastovo, the list of plants used must already have been quite short a few decades ago, but on some islands such as Brač, Šolta, Vis or Pašman, 

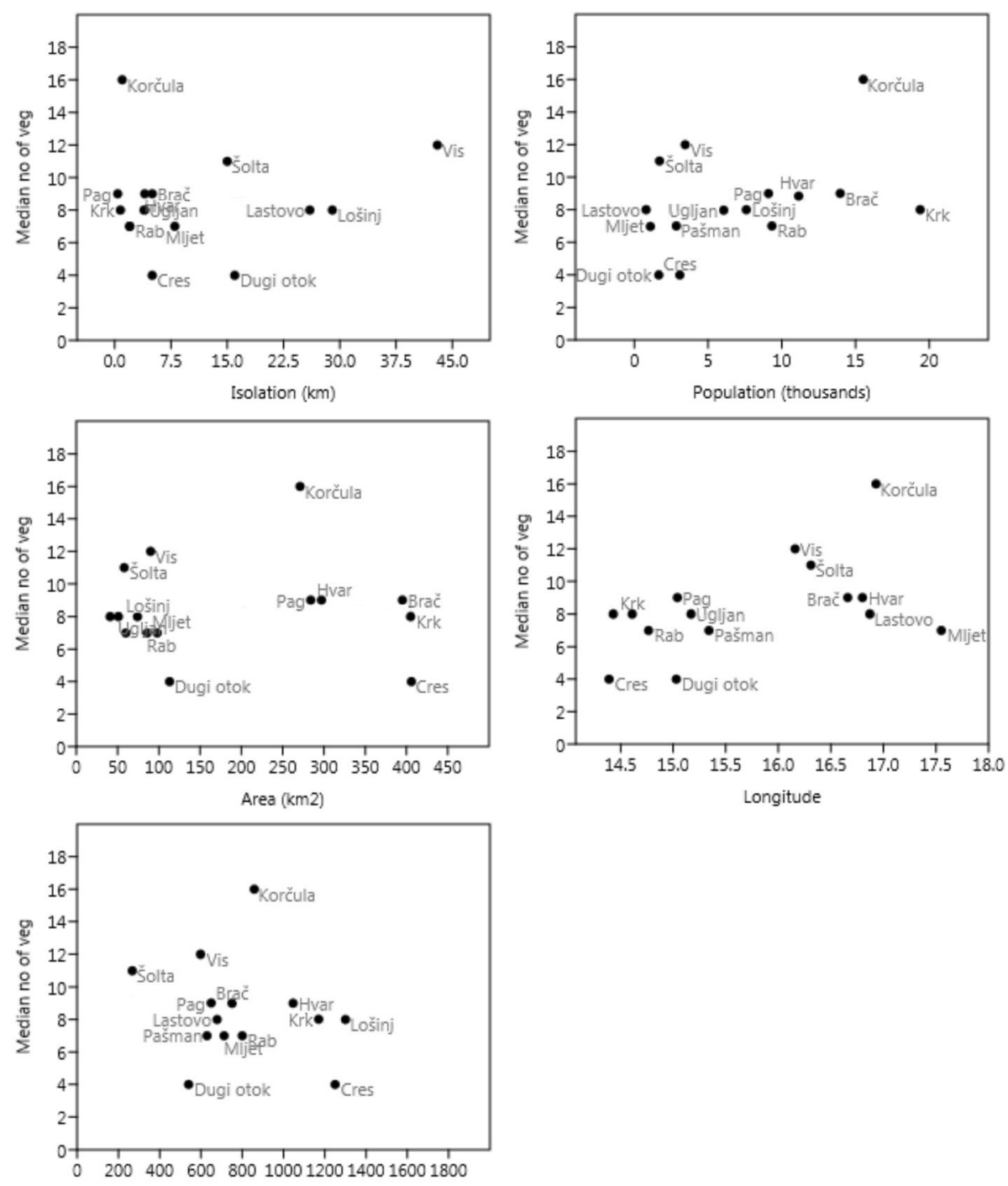

Flora

Fig. 2 Scatterplots of the median numbers of vegetables used and the studied independent variables

Table 4 The correlation matrix of all the variables in the study (correlation coefficients in the lower left half, $P$ values in the upper right half). Most correlations are expressed as Pearson $r$ coefficient. Only correlations for area (printed in italics) were calculated using Spearman rs rank correlation coefficient (see explanation in the 'Methods' section)

\begin{tabular}{|c|c|c|c|c|c|c|c|}
\hline & No. of veg & Median no. of veg & Area $^{a}$ & Population & Flora & Longitude & Isolation \\
\hline No. of veg & & $2.6 \mathrm{E}-05$ & 0.75 & 0.21 & 0.37 & 0.11 & 0.57 \\
\hline Median no. of veg & 0.87 & & 0.96 & 0.15 & 0.48 & 0.06 & 0.67 \\
\hline Area $^{a}$ & 0.09 & -0.01 & & -0.03 & 0.07 & 0.45 & 0.15 \\
\hline Population & 0.34 & 0.39 & 0.55 & & 0.09 & 0.73 & 0.01 \\
\hline Flora & -0.26 & -0.21 & 0.49 & 0.47 & & 0.14 & 0.55 \\
\hline Longitude & 0.42 & 0.50 & -0.17 & -0.10 & -0.42 & & 0.47 \\
\hline Isolation b & -0.15 & -0.12 & -0.39 & -0.63 & -0.18 & 0.20 & \\
\hline
\end{tabular}

${ }^{\mathrm{a}}$ For this variable, Spearman $r$ s (rank correlation) coefficient was calculated

${ }^{\mathrm{b}}$ This variable was log-transformed in order to achieve normal distribution 


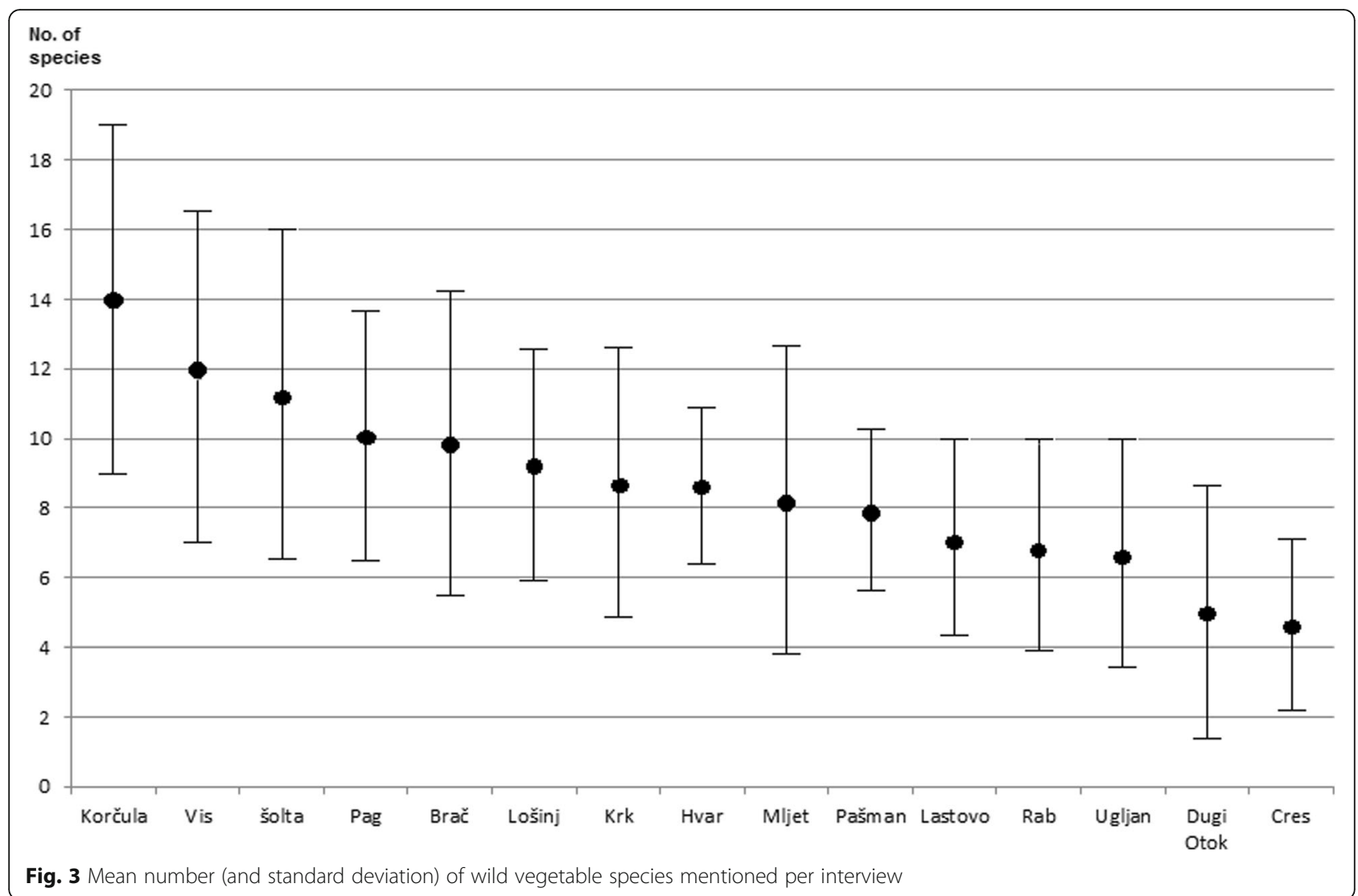

the collecting of wild vegetables was widespread even at the end of the twentieth century and collapsed quite recently, with several older people still practicing it now. It is only Korčula where the custom is important even nowadays, although signs of the deterioration of knowledge and a reduction in the number of collected species are visible even there. The differences between islands cannot be explained based on apparency [40] or resource availability theory [41] of the main species of wild vegetables as they are common and easily found on

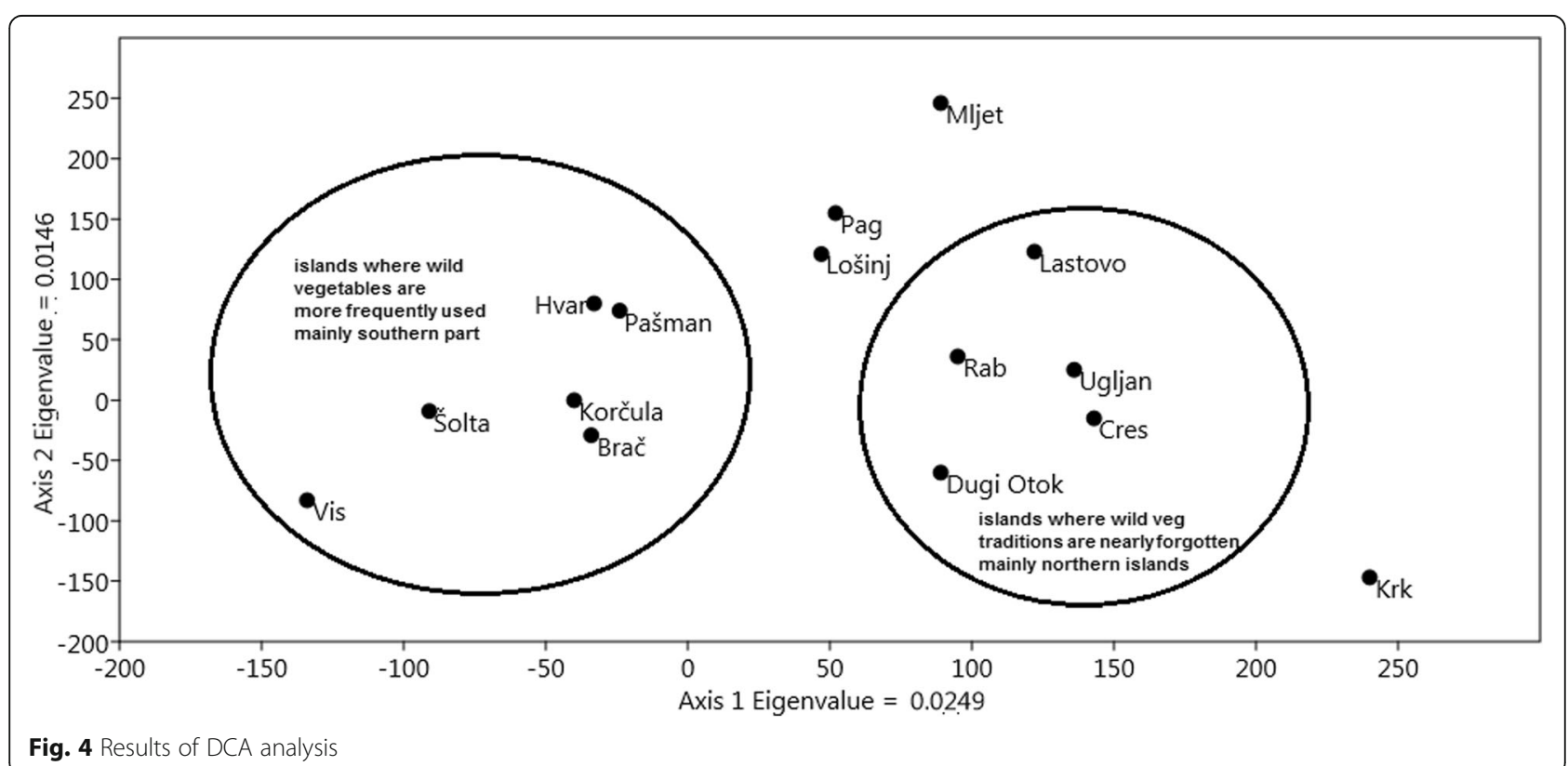




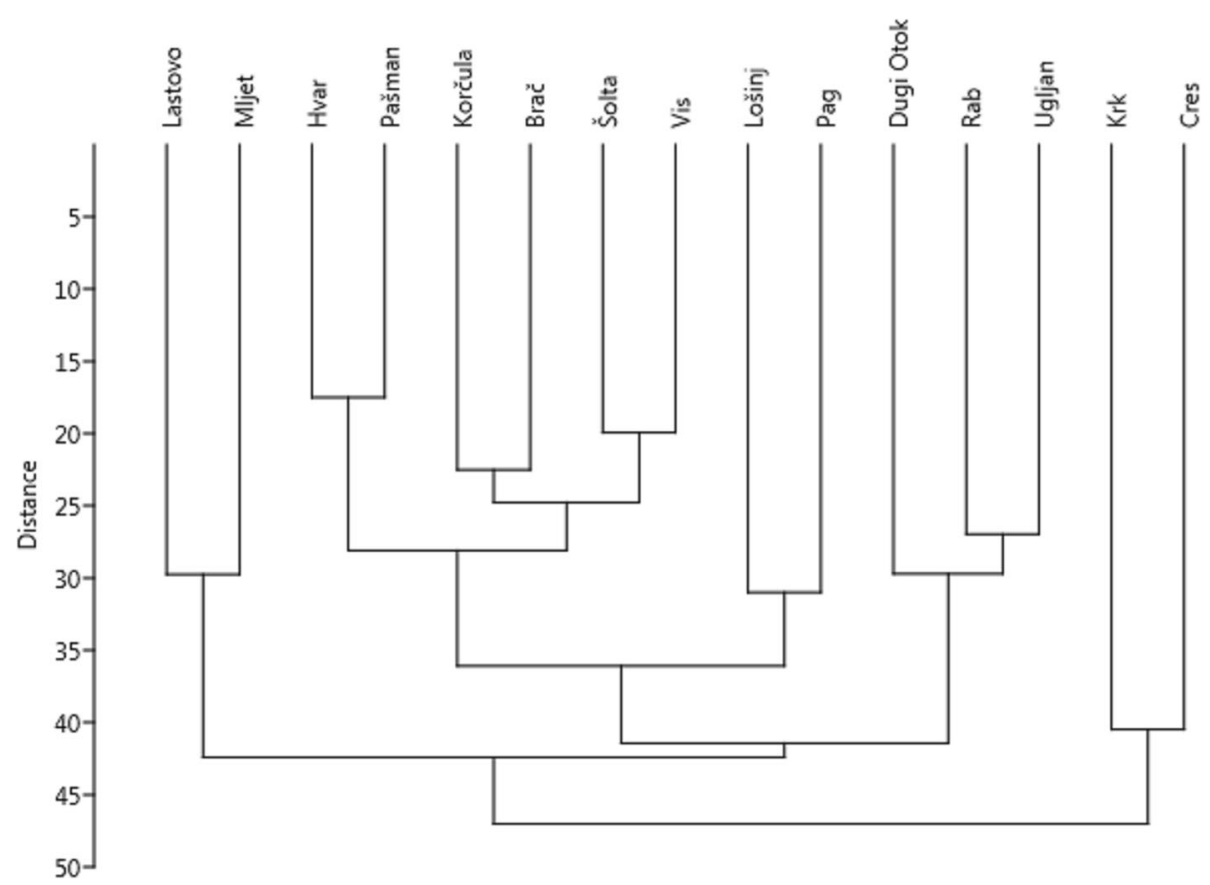

Fig. 5 The dendrogram of UPGMA clustering of islands based on the matrix of wild vegetables used in them

each island. They are probably caused mainly by the differences in the rate of abandonment of the old tradition of eating wild vegetables. The gradual abandonment of using wild vegetables has been observed in other Mediterranean regions of Europe (see e.g. [26, 42]), and it is only recently that some health-conscious people and those interested in cooking have gone back to it $[17,42]$. We recorded only two species for which some of our respondents observed a positive trend. One of them is
Asparagus acutifolius. Around 30-50 years ago, in some villages, it was not collected, but the use of this species spread to most families. Many informants attribute this increase in consumption to the cessation of grazing by livestock and a consequent increase in the populations of $A$. acutifolius in the wild. The other is $C$. maritimum which was collected in the past but to a much lesser extent. Its current widespread use has been popularised both by TV cooking programmes and by people from

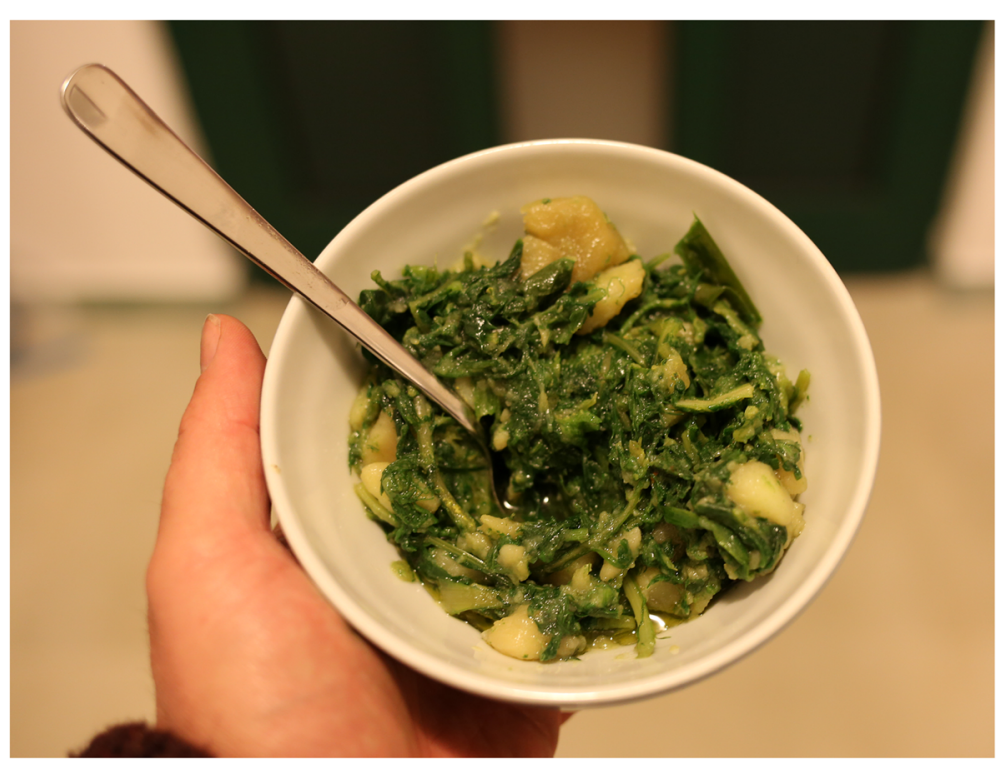

Fig. 6 A bowl of gruda, i.e. wild vegetable mix from Vela Luka, Korčula, cooked with potatoes and spiced with olive oil and salt 
outside the community being seen to collect it. C. maritimum is now commonly sold preserved in vinegar as a souvenir for tourists.

Why has the population of Korčula preserved the largest number of wild vegetables used? Korčula was spotted as a place with a rich tradition of using wild vegetables as far back as 1981, when a TV programme broadcast by 'Radiotelevision Zagreb' was made (after [25]). An article about the tradition was also written by a local museum worker [43], and a book about plant uses was compiled by a local school teacher together with her primary school students [44]. Long lists of wild vegetables used also occur in the local dialect dictionary [45] and in a monograph of the island [46]. No other studied island has produced such publications or such a strong local identification with using wild vegetables. There may be another reason for the very robust knowledge of wild vegetables in Korčula. The island has always had a large population which maintains its subsistence on cultivated crops. In the early twentieth century, Korčula experienced a very severe famine, as a consequence of the mass destruction of vineyards caused by a phylloxera epidemic (information from older informants). In contrast to this, less populated and more isolated islands such as Dugi Otok and Lastovo could base their nutritional economy on marine resources and were not affected by malnutrition-the latter also served as a smuggling base, which brought high cash profits.

The list of wild vegetables used is very typical for the Mediterranean areas of Europe [15, 16, 26, 42, 47-56]. It is also similar to those recorded in other parts of Croatia [19-24].

Some of the islands, especially in the north-western half of the study area (Kvarner and Zadar archipelago), contain large salt-marshes. Surprisingly, the only typically coastal halophilous plant widely utilised in the Adriatic Islands is C. maritimum. We only found a few respondents using wild Beta vulgaris L. (on various islands throughout), Sarcocornia fruticosa (L.) A.J.Scott. (only on Rab and Lošinj) and Salsola soda L. (on Pag and some smaller islands between Zadar and Split not included in this study). Our data show that coastal areas were treated as sources of food for animal stock, rather than sources of plant food for humans. C. maritimum is now widely collected for pickles, but in most cases, this is a new fashion which people took up a few years ago, though a certain proportion of informants remember making such pickles in their childhood as well. The lack of food use of sea marsh plants is particularly striking on Pag, famous for this type of vegetation.

Out of the recorded genera, we have not found any whose use is specific only to the Adriatic Islands apart from seagrass (Posidonia oceanica (L.) Delile). The basal parts of the shoots of this monocot species were used to be eaten as a snack on Korčula and Vis. Unfortunately, no traces of the traditional use of seaweeds have been recorded. Another interesting find was the custom of eating raw tubers of Bunium alpinum Waldst. \& Kit. s.l. on Pašman and Brač. We have not found any other food uses of these two species in world literature, though other Bunium species are widely known to be used as food. For example, in Spain, three species are used, Bunium balearicum (Sennen) Mateo \& López Udías, Bunium macuca Boiss and Bunium pachypodum P.W. Ball [40]. Yet, another interesting tradition is eating the flowering shoots of the parasitic Cytinus hypocistis (L.) L., which is still widely known (though its practice ceased a few decades ago) on the island of Pašman. The consumption of $C$. hypocystis was reported before only from small localities in Spain, Portugal, Turkey and Greece [46-50].

Vis is the island with the second longest list of wild vegetables used. What differentiates it from other islands is the custom of eating young shoots and green pods of a few Fabaceae plants, with the immature fruits of Lotus edulis L. particularly prized as a raw snack or for pickling (like capers).

\section{Conclusions}

The recorded relationships between the demographic and geographical features of islands were weak and statistically not significant. It is most likely that cultural and historical factors diversifying the use of plants in particular islands are stronger than the above-mentioned quantitatively measurable variables. A general trend of increasing richness in wild vegetables from north-west to south-east (which can have cultural or historical reasons) can be observed. More ethnobotanical quantitative studies on islands are needed to form an 'island ethnobotany theory'.

\section{Acknowledgments \\ Our special thanks go to all the study participants who wanted to share their knowledge. \\ Funding \\ The research was financed by funds from the Ministry of Science and Higher Education, the National Science Centre in Poland (NCN) [2015/19/B/HS3/ 00471].}

\section{Availability of data and materials}

The data matrix analysed during the current study is available upon request. Voucher specimens for species were deposited in the herbariums of Warsaw University (WAW) and the University of Zagreb (ZAGR).

\section{Authors' contributions}

Ł contributed to the concept of the study and the first draft of the paper. All the authors read and approved the final version of the paper and took part in the field study including interviewing and voucher specimen collection: $Ł Ł$ (14 islands), IVK (9), MJD (5), KD (2) and MJ (1). 


\section{Ethics approval and consent to participate}

The research adhered to the local traditions for such research, the Code of Ethics of the International Society of Ethnobiology [57] and the American Anthropological Association Code of Ethics (2009) [58]. Prior oral informed consent was obtained from all study participants. No ethical committee permits were required. No permits were required to collect voucher specimens.

\section{Consent for publication}

Not applicable.

\section{Competing interests}

The authors declare that they have no competing interests.

\section{Publisher's Note}

Springer Nature remains neutral with regard to jurisdictional claims in published maps and institutional affiliations.

\section{Author details}

'Department of Botany, Faculty of Biotechnology, University of Rzeszów, ul. Pigonia 1, 35-310 Rzeszów, Poland. Institute for Adriatic Crops and Karst Reclamation, Put Duilova 11, 21000 Split, Croatia. ${ }^{3}$ Institute for Marine and Coastal Research, University of Dubrovnik, Kneza Damjana Jude 12, PO Box 83, 20000 Dubrovnik, Croatia. ${ }^{4}$ Žrnovo, Croatia. ${ }^{5}$ Department of Agricultural Botany, Faculty of Agriculture, University of Zagreb, Svetošimunska cesta 25 10000 Zagreb, Croatia.

\section{Received: 4 October 2018 Accepted: 8 March 2019}

Published online: 29 March 2019

\section{References}

1. Darwin C, Wallace A. On the tendency of species to form varieties; and on the perpetuation of varieties and species by natural means of selection. J Proc Linn Soc London Zool. 1858;3(9):45-62.

2. MacArthur RH, Wilson EO. The theory of island biogeography. Princeton University Press: Princeton; 1967.

3. Figueiredo GM, Leitao-Filho HF, Begossi A. Ethnobotany of Atlantic Forest coastal communities: diversity of plant uses in Gamboa (Itacuruçá Island, Brazil). Hum Ecol. 1993;21:419-30.

4. Thaman RR. Ethnobotany of Pacific Island coastal plants. Sci Pac Island Peoples: Fauna, Flora, Food Med. 1994;3:147-84

5. McClatchey WC. Wild food plants of Remote Oceania. Acta Soc Bot Pol. 2012;81:371-84

6. Quave $\mathrm{CL}$, Pieroni A. A reservoir of ethnobotanical knowledge informs resilient food security and health strategies in the Balkans. Nat Plants. 2015; $1: 14021$

7. Rudan P, Šimić D, Smolej-Narančić N, Bennett LA, Janićijević B, Jovanović V, Lethbridge MF, Miličić J, Roberts DF, Sujoldžíc A, Szirovicza L. Isolation by distance in Middle Dalmatia_Yugoslavia. Am J Phys Anthropol. 1987;74(3): 417-26.

8. Rudan I, Rudan D, Campbell H, Carothers A, Wright A, Smolej-Narancic N, Janicijevic B, Jin L, Chakraborty R, Deka R, Rudan P. Inbreeding and risk of late onset complex disease. J Med Genet. 2003:40(12):925-32.

9. Šarac J. The remote isolate of the Croatian littoral-50 years of research on the island of Susak with new insights into its genetic diversity. Coll Antropol. 2017:41(1):1-0.

10. Vitart V, Biloglav Z, Hayward C, Janićijević B, Smolej-Narančić N, Barać L, Peričić M, Martinović-Klarić I, Škarić-Jurić T, Barbalić M, Polašek O, Kolčić I, Carothers A, Rudan P, Hastie N, Wright A, Campbell H, Rudan I. 3000 years of solitude: extreme differentiation in the island isolates of Dalmatia, Croatia. Eur J Hum Gen. 2006;14:478-87.

11. Ostroški L, editor. Statistički ljetopis Republike Hrvatske 2015, Statistical Yearbook of the Republic of Croatia 2015. Zagreb: Croatian bureau of statistics; 2015. http://wwwdzshr/Hrv_Eng/ljetopis/2015/sljh2015pdf Accessed 27 Aug 2018

12. Nejašmić I, Mišetić R. Migration and population decline in the island of Vis, Croatia 1910-2001. Assoc Eur Migr Inst AEMI J. 2006:4:107-25.

13. Nikolić T, Antonić O, Alegro AL, Dobrović I, Bogdanović S, Liber Z, Rešetnik I. Plant species diversity of Adriatic islands: an introductory survey. Plant Biosyst. 2008;142:435-45.
14. Pandža M, Milović M. Floristic researches of the island of Pašman, Croatia. In: Škvorc Ž, Franjić J, editors. 36th Meeting of Eastern Alpine and Dinaric Society for Vegetation Ecology - Book of Abstracts. Zagreb: Eastern Alpine and Dinaric Society for Vegetation Ecology; 2015. p. 43.

15. Hadjichambis AC, Paraskeva-Hadjichambi D, Della A, Giusti EM, De Pasquale C, Lenzarini C, Censorii E, Reyes Gonzales-Tejero M, Patricia Sanchez-Rojas C, Ramiro-Gutierrez JM, Skoula M. Wild and semi-domesticated food plant consumption in seven circum-Mediterranean areas. Int J Food Sci Nutr. 2008;59(5):383-414.

16. Rivera D, Obon C, Heinrich M, Inocencio C, Verde A, Fajardo J. Gathered Mediterranean food plants-ethnobotanical investigations and historical development. Local Mediterranean Food Plants Nutraceutic. 2006;59:18-74.

17. Łuczaj Ł, Pieroni A, Tardío J, Pardo-de-Santayana M, Sõukand R, Svanberg I, Kalle R. Wild food plant use in 21 st century Europe, the disapperance of old traditions and the search for new ciusines involving wild edibles. Acta Soc Bot Pol. 2012:81:359-70.

18. de Cortes S-MM, Tardío J, editors. Mediterranean wild edible plants: ethnobotany and food composition tables. New York: Springer; 2016.

19. Łuczaj Ł, Zvonko Končić M, Miličević T, Dolina K, Pandža M. Wild vegetable mixes sold in the markets of Dalmatia (southern Croatia). J Ethnobiol Ethnomed. 2013:9:2

20. Łuczaj Ł, Fressel N, Perković S. Wild food plants used in the villages of the Lake Vrana Nature Park (northern Dalmatia, Croatia). Acta Soc Bot Pol. 2013; 82:275-81.

21. Dolina K, Jug-Dujaković M, Łuczaj Ł, Vitasović-Kosić I. A century of changes in wild food plant use in coastal Croatia: the example of Krk and Poljica. Acta Soc Bot Pol. 2016;85. https://doi.org/10.5586/asbp.3508.

22. Dolina K, Łuczaj $Ł$. Wild food plants used on the Dubrovnik coast (southeastern Croatia). Acta Soc Bot Pol. 2014;83:175-81.

23. Łuczaj $Ł$, Dolina K. A hundred years of change in wild vegetable use in southern Herzegovina. J Ethnopharmacol. 2015;166:297-304.

24. Vitasović-Kosić I, Juračak J, Łuczaj Ł. Using Ellenberg-Pignatti values to estimate habitat preferences of wild food and medicinal plants: an example from northeastern Istria (Croatia). J Ethnobiol Ethnomed. 2017:13:31.

25. Jug-Dujaković M, Łuczaj $Ł$. The contribution of Josip Bakić's research to the study of wild edible plants of the Adriatic coast: a military project with ethnobiological and anthropological implications. Slovak Ethnol. 2016;64: 158-68.

26. Biscotti N, Pieroni A. The hidden Mediterranean diet: wild vegetables traditionally gathered and consumed in the Gargano area, Apulia, SE Italy. Acta Soc Bot Pol. 2015:84:327-39.

27. Leonti M. The future is written: impact of scripts on the cognition, selection, knowledge and transmission of medicinal plant use and its implications for ethnobotany and ethnopharmacology. J Ethnopharmacol. 2011;134:542-55.

28. Martin GJ. Ethnobotany: a methods manual. London: Chapman \& Hall; 1995.

29. Alexiades MN, Sheldon JW. Selected guidelines for ethnobotanical research: a field manual. New York: New York Botanical Garden; 1996.

30. Albuquerque UP, Alves RR, editors. Introduction to ethnobiology. New York: Springer; 2016.

31. Anderson EN, Pearsall D, Hunn E, Turner N, editors. Ethnobiology. New York: Wiley; 2012.

32. Domac R. Flora Hrvatske: priručnik za određivanje bilja. Zagreb: Školska knjiga; 1994.

33. Pignatti S. Flora d'Italia. Edagricole: Bologna; 1982.

34. Flora Croatica Database. https://hirc.botanic.hr/fcd/ Accessed 15 Jan 2019.

35. The Plant List. http://www.theplantlist.org. Accessed 15 Jan 2019.

36. Hammer $\varnothing$, Harper DAT, Ryan PD. PAST: paleontological statistics software package for education and data analysis. Palaeontol Electron. 2001;4:9.

37. Hill MO, Gauch HG. Detrended correspondence analysis: an improved ordination technique. In: der Maarel v, editor. Classification and Ordination Symposium on advances in vegetation science, Nijmegen, The Netherlands, May 1979. Dordrecht: Springer; 1980. p. 47-58.

38. Sokal R, Michener CA. Statistical method for evaluating systematic relationships. University of Kansas Science Bulletin. 1958:38:1409-38.

39. Bailey K. Typologies and taxonomies: an introduction to classification techniques. London-New Delhi: Sage Publications; 1994.

40. Feeny PP. Plant apparency and chemical defense. In: Wallace JW, Mansell $\mathrm{RL}$, editors. Recent advances in phytochemistry. New York: Plenum Press; 1976. p. 1-40.

41. Coley PD, Bryant JP, Chapin FS. Resource availability and plant antiherbivore defense. Science. 1985;230:895-9. 
42. Reyes-García V, Menendez-Baceta G, Aceituno-Mata L, Acosta-Naranjo R, Calvet-Mir L, Domínguez P, Garnatje T, Gómez-Baggethun E, MolinaBustamante M, Molina M, Rodríguez-Franco R. From famine foods to delicatessen: interpreting trends in the use of wild edible plants through cultural ecosystem services. Ecol Econ. 2015;120:303-11.

43. Sardelić S. Samoniklo jestivo bilje - mišanca, gruda, parapač. Etnološka Istraživanja. 2008;1 (12/13):387-96.

44. Skokandić A, Cebalo A, Graber T, Laus M, Matulović V, Stanarević N, Vranješ M. Vratimo se prirodi. Žrnovo: Osnovna škola "Ante Curać - Pnjac" in Žrnovo; 2013.

45. Baničević B. Rječnik starinskih riječi u Smokvici na Korčuli. 2nd ed. Smokvica: self-published; 2016.

46. Gjivoje M. Otok Korčula. 2nd ed. Zagreb: self-published; 1969.

47. Geraci A, Amato F, Di Noto G, Bazan G, Schicchi R. The wild taxa utilized as vegetables in Sicily (Italy): a traditional component of the Mediterranean diet. J Ethnobiol Ethnomed. 2018;14:14.

48. Tardío J, Pascual H, Morales R. Wild food plants traditionally used in the province of Madrid, Central Spain. Plantas silvestres alimentarias usadas tradicionalmente en la provincia de Madrid, centro de España. Econ Bot. 2005;59(2):122.

49. Bulut G. Medicinal and wild food plants of Marmara Island (Balikesir-Turkey). Acta Soc Bot Pol. 2016;85(2):3501.

50. Ertuğ F. Wild edible plants of the Bodrum area (Muğla, Turkey). Turk J Bot. 2004;28(1-2):161-74

51. Rivera D, Obón C, Inocencio C, Heinrich M, Verde A, Fajardo J, Palazón JA. Gathered food plants in the mountains of Castilla-La Mancha (Spain): ethnobotany and multivariate analysis. Econ Bot. 2007;61(3):269.

52. Carvalho AM, Morales R. Persistence of wild food and wild medicinal plant knowledge in a North-Eastern region of Portugal, Ethnobotany in the New Europe: People, health and wild plant resources; 2010. p. 147-71.

53. Ciftcioglu GC. Sustainable wild-collection of medicinal and edible plants in Lefke region of North Cyprus. Agrofor Syst. 2015;89(5):917-31.

54. Forbes MH. Farming and foraging in prehistoric Greece: a cultural ecological perspective. Ann N Y Acad Sci. 1976;268(1):127-42.

55. Leonti M, Nebel S, Rivera D, Heinrich M. Wild gathered food plants in the European mediterranean: a comparative. Econ Bot. 2006;60(2):130-42.

56. Cucinotta F, Pieroni A. "If you want to get married, you have to collect virdura": the vanishing custom of gathering and cooking wild food plants on Vulcano, Aeolian Islands, Sicily. Food, Culture Soc. 2018;21(4):539-67.

57. International Society of Ethnobiology Code of Ethics (with 2008 additions). http://ethnobiology.net/code-of-ethics. Accessed 10 Feb 2017.

58. American Anthropological Association Code of Ethics. http://www.aaanet. org/issues/policy-advocacy/upload/AAA-Ethics-Code-2009.pdf. Accessed 10 Feb 2017.

Ready to submit your research? Choose BMC and benefit from:

- fast, convenient online submission

- thorough peer review by experienced researchers in your field

- rapid publication on acceptance

- support for research data, including large and complex data types

- gold Open Access which fosters wider collaboration and increased citations

- maximum visibility for your research: over $100 \mathrm{M}$ website views per year

At BMC, research is always in progress.

Learn more biomedcentral.com/submissions 